\title{
ANALYSIS OF SERVICE BROKER POLICIES IN CLOUD ANALYST FRAMEWORK
}

\author{
Ashish Sankla ${ }^{1 *}$ \\ ${ }^{* 1}$ G. B Pant Govt. Engineering College, Computer Science Department, New Delhi, India \\ Sanklaon31st@gmail.com
}

\section{*Corresponding Author: -}

E-mail ID - Sanklaon31st@gmail.com

\begin{abstract}
: -
The aim of the paper is to analyze different service broker policies that can be used with cloud and are been used in cloud analyst. With the advancement in the cloud computing technologies cloud is becoming more popular these days. With the increase in the number of clients and servers there is also arising needs of service broker. Broker is been used to assign a server to the client on clients request. Here I will first discuss about cloud computing, and then cloud analyst and the different broker policies embedded in it under round robin load balancing policy. Then with the help of simulation results will propose the best broker policy on the basis of performance.
\end{abstract}

Index Terms: - Cloud Computing, Broker, Service Broker, Cloud Analyst, Broker Policies.

\section{(c) (\$) (1)}




\section{INTRODUCTION}

A rapid advancement is been viewed in the area of Cloud computing. This technology is becoming quite popular among the industry as well as academics [4]. It is emerging as one of the most challenging and promising technology. Cloud technology aims at offering elastic, distributed and virtualized resources as utility to end users and also supports realization of "computer as a utility" [1]. Also with the advancement of cloud there are new possibilities been generated for the developers for internet based applications development and emerging.

\section{A. Types of Cloud}

There are five types of Cloud on the basis of deployment [3]:

- Public Cloud: IT services provided by cloud can be publically used by anyone using internet.

- Private Cloud: IT services are only provided to a group of authorized users like an enterprise. The cloud service provider can be an internal IT organization or a third party.

- Hybrid Clouds: Environment is created through the usage of private and public cloud offerings by an organization.

- Internal Cloud: Environment is a subset of a private cloud model. Here the cloud environment is the service provided by the same organization to its sub part.

- External Clouds: Environment hosted by other IT organization and offered as a service to another outside IT organization. It is implemented by a third party and environment can be public or private.

\section{B. Define of Cloud}

"Cloud Computing refers as applications delivery as service over the Internet and system software and hardware in the datacenters that provides those services" [6].

\section{Technological Development in Cloud}

The Cloud is based on two separate technological developments [1]:

\section{- Cloud Service Providers:}

These are service providers for cloud willing to provide their services i.e. large scale computing infrastructure at cost based on usage pattern. It eliminates the initial high-cost investment on part of developer to create an environment for application development.

\section{- Software System Providers:}

They develop applications as e-commerce and social networking sites, which are gaining popularity these days on internet. These types of applications are of great benefit to cloud infrastructure service quality to improve service quality and to minimize costs.

For developers who wants to develop such applications require acquisition of servers those are capable of handling the peak demands, installation of applications, whole software infrastructure and configuration of the application itself. And can also choose different cloud environment as per user requirements.

The maximum servers remain underutilized as there are fixed peak traffic timings. This problem is overcome by payper-usage method used by cloud service providers. Cloud Broker handles the traffic routing between data centers and user bases.

Cloud service broker creates a secure cloud management platform. This platform is to ease and simplify the delivery of complex cloud services to users of cloud services. It enables users to realize and experience the full potential that a cloud service provider offers by creating a secure, governed and trusted cloud management platform between the user and the service provider.

In this paper a tool named as Cloud Analyst is used. It supports simulation and visual modeling of large scale application deployed on cloud. Cloud Analyst is a tool built on Cloud Sim framework. It allows user to add description of application, geographic location of user and data centers, number of users and data centers, resource allocation for each data center, peak usage hours as per geographical location, broker policy, and load balancing policy. Using this information Cloud Analyst generates result about request response time, processing time, and cost as per data usage and other metrics.

By using Cloud Analyst I determine the best broker policy with respect to performance.

\section{Cloud analyst}

Deployment of large-scale applications on Cloud is easier and cheaper. As cloud applications can be deployed in several geographical locations and it also affects the user located far away from the datacenter. Users are accessing internet applications from around the world and the popularity of the application also varies with geographical location and so will vary the experience of user of using that application.

The impact of simultaneous quantifying number of users, geographical location of relevant component and network in application is hard to achieve in real testbeds, as the presence of such elements cannot be controlled nor predicted by developers.

Cloud Analyst is a tool that gives solution to the above problems by allowing user to configure the simulator by its userfriendly graphical user interface and configure the duration of simulation, number of users as per their geographical location, data centers and their configuration, and choose from the three different service broker polices. 


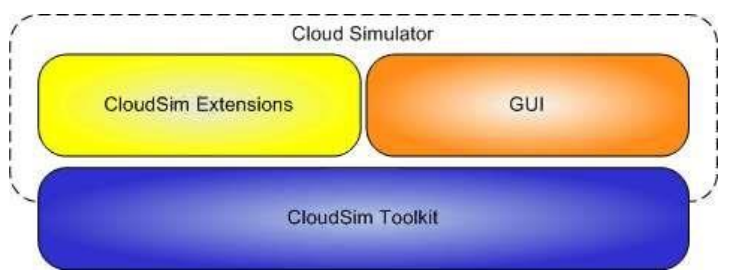

Fig 1: Cloud Analyst Architecture [1].

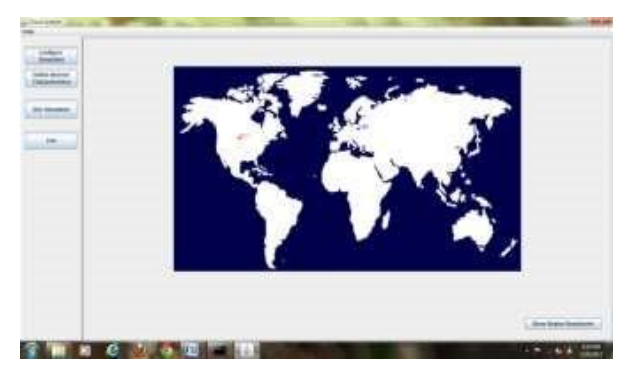

Fig 2: Cloud Analyst Simulation Screen.

\section{Service broker}

A data-intensive Cloud environment can be compared to the real world economic system wherein there are consumers and producers of data. Producers are those who generate the data and control its distribution by copying replica around the globe at different locations. The criteria such as storage and computational requirements, min. bandwidth and access restriction, security and data locality issues should also be considered [5].

\section{A. Broker}

A broker performs discovery of resource based on characteristics defined by user. It also identifies the list of data sources and replicas available and chooses the optimal one. The broker also ensures minimum credit required by the user to access the resources. It collects the results and passes them to the user.

\section{B. Broker Policies}

In Cloud Analyst there are three service broker policies available each implementing different routing policy

- Closest Data Center: It is based on network latency; proximity is the fastest path to the data center from a user base. Broker will route user traffic to the closest data center.

- Optimise Response Time: It actively monitors the performance of all data centers and directs traffic to that data center which estimates to give the least response time.

- Reconfigure Dynamically: It is an experimental service broker policy for peak load sharing. The

load is been shared by allocating virtual machines to the data centers according to the best processing time as compared with the current processing time.

\section{Configuring simulation}

Here six user bases are created representing the six different regions. Each user base is contained in single time zone and assumption is been taken about the peak hours and online users during the peak hours. For this assumption randomly 2 consecutive hours in the evening are taken as peak hours and the user base is been reduced to $1 \%$ and then $10 \%$ of users are assumed to be online during peak hours and $1 \%$ of the users are been considered online during off peak hours. Eg:According to [7] the total number of users

\section{A. Simulator Configuration}

Table 1: Region wise number of users of FaceBook

\begin{tabular}{|l|l|l|}
\hline $\begin{array}{l}\text { Region } \\
\text { Cloud Analyst }\end{array}$ & Region id & Users in millions \\
\hline North America & 0 & 168 \\
\hline South America & 1 & 115 \\
\hline Europe & 2 & 209 \\
\hline Asia & 3 & 153 \\
\hline Africa & 4 & 3 \\
\hline Oceanic & 5 & 12 \\
\hline
\end{tabular}

Rest configurations as per [1].

The simulation is been configured and executed for two broker policies i.e.

- Closest Data Center

- Optimise Response time

and they both are considered under Round Robin load balancing policy. 
The scenarios on which the result is based are described in the following table and all the further referencing and comparison is been done under the reference number

Table 2: Scenarios and their Description

\begin{tabular}{|l|l|}
\hline Scenario & Scenario Description \\
\hline 1 & 1 Data Center(DC) 50 Virtual machines(VMs) \\
\hline 2 & 2 DCs with 25 VMs each \\
\hline 3 & 2 DCs with 50 VMs each \\
\hline 4 & 3 DCs with 50 VMs each \\
\hline 5 & 3 DCs with 75, 50 and 25 VMs \\
\hline
\end{tabular}

\section{B. Simulation Results}

The result is further sub divided in two parts on the basis of service broker policy been used.

\section{a) Closest Data Center}

Table 3: Overall Response Time

\begin{tabular}{|l|l|l|l|}
\hline Scenario & Avg.(ms) & Min.(ms) & Max.(ms) \\
\hline 1 & 300.20 & 42.28 & 639.63 \\
\hline 2 & 8628.83 & 97.83 & 31956.61 \\
\hline 3 & 4296.92 & 72.97 & 20154.00 \\
\hline 4 & 4404.73 & 63.97 & 13324.59 \\
\hline 5 & 4523.02 & 64.83 & 18519.58 \\
\hline
\end{tabular}

Table 4: Data Center Processing/Request Servicing Time

\begin{tabular}{|l|l|l|l|}
\hline Scenario & Avg.(ms) & Min.(ms) & Max.(ms) \\
\hline 1 & 19.12 & 0.11 & 98.38 \\
\hline 2 & 8414.22 & 10.09 & 31896.46 \\
\hline 3 & 4116.30 & 10.09 & 20090.20 \\
\hline 4 & 4294.96 & 10.09 & 13266.97 \\
\hline 5 & 4404.34 & 10.09 & 18519.07 \\
\hline
\end{tabular}

Table 5: Total Expenses

\begin{tabular}{|l|l|l|l|}
\hline Scenario & VMs \$ & $\begin{array}{l}\text { Date } \\
\text { Transfer \$ }\end{array}$ & Total \$ \\
\hline 1 & 120.05 & 325.48 & 445.53 \\
\hline 2 & 120.05 & 325.48 & 445.53 \\
\hline 3 & 240.10 & 325.48 & 565.58 \\
\hline 4 & 360.15 & 351.96 & 712.11 \\
\hline 5 & 360.15 & 325.48 & 685.63 \\
\hline
\end{tabular}

b) Optimise Response Time

Table 6: Overall Response Time

\begin{tabular}{|l|l|l|l|}
\hline Scenario & Avg.(ms) & Min.(ms) & Max.(ms) \\
\hline 1 & 300.21 & 41.83 & 669.84 \\
\hline 2 & 7474.32 & 75.20 & 32220.48 \\
\hline 3 & 3679.76 & 62.13 & 13327.37 \\
\hline 4 & 3341.03 & 59.54 & 12877.93 \\
\hline 5 & 3111.52 & 61.22 & 12879.67 \\
\hline
\end{tabular}


Table 7: Data Center Processing/Request Servicing Time

\begin{tabular}{|l|l|l|l|}
\hline Scenario & Avg.(ms) & Min.(ms) & Max.(ms) \\
\hline 1 & 19.07 & 0.11 & 98.78 \\
\hline 2 & 7225.90 & 10.01 & 32158.53 \\
\hline 3 & 3461.96 & 10.01 & 13269.95 \\
\hline 4 & 3156.93 & 10.01 & 12817.65 \\
\hline 5 & 2932.73 & 10.01 & 12554.63 \\
\hline
\end{tabular}

Table 8: Total Expenses

\begin{tabular}{|l|l|l|l|}
\hline Scenario & Avg.(ms) & Min.(ms) & Max.(ms) \\
\hline 1 & 120.05 & 325.48 & 445.53 \\
\hline 2 & 120.05 & 325.48 & 445.53 \\
\hline 3 & 240.10 & 325.48 & 565.58 \\
\hline 4 & 360.15 & 325.48 & 685.63 \\
\hline 5 & 360.15 & 325.48 & 685.63 \\
\hline
\end{tabular}

\section{OBSERVATIONS}

A. Observations on Basis of Simulation Results

- The overall response time for table 6 is less as compared to table 3 for all the scenarios except scenario 1 max response time.

- There is a gradual decrease in the overall response time of table 6 with the increase in the number of data centers and virtual machines.

- The request service time of table 7 is comparatively less than that of table 4 .

- All the scenarios have shown the monotonic downfall in request time in table 7 with the increase in the number of data centers and virtual machine.

- The virtual machine and data transfer cost is same for both the cases.

\section{CONCLUSION}

It is clearly visible by the above data the "Optimize response time" broker policy approach is the best in the cloud analyst frame work and that can be further supported by the charts below to show the comparison between the average response time using "Optimise response time" broker and "Closest data center" broker policy.

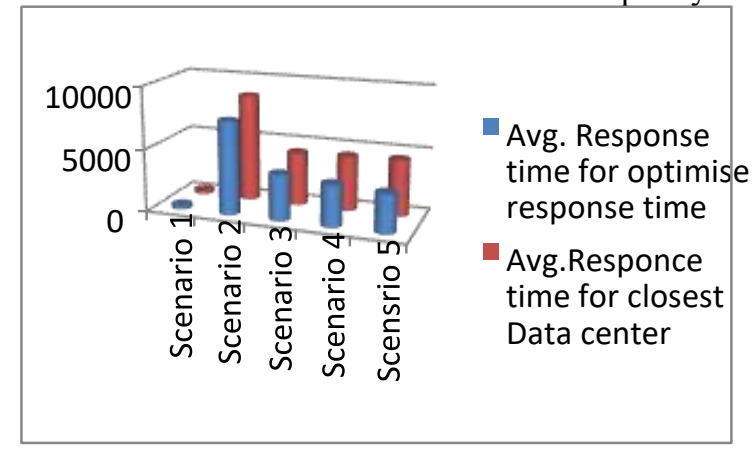

Chart 1: Comparing Avg. Response Time.

In chart 1 the difference is visible clearly in the average response time.

The Average time required for servicing a request or the data center processing time is less for the "Optimise response time" broker policy as compared to other broker policy.

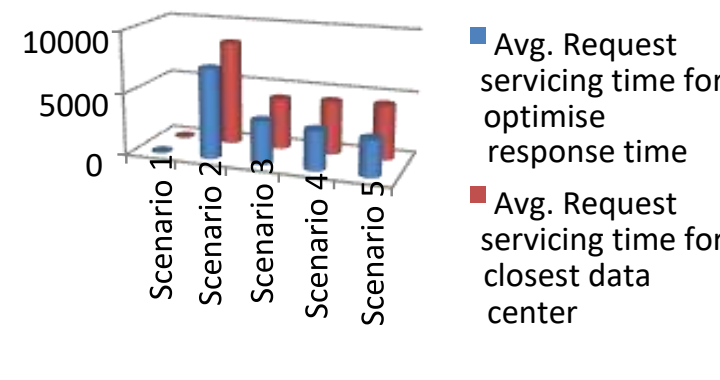

Chart 2: Comparing Avg. Request Servicing Time. 
So, it is clear on the basis of comparison the "Optimise Response Time" broker policy is more efficient on the basis of its performance.

\section{REFERENCES}

[1]. B. Wickremasinghe, Rodrigo N. Calheiros, and Rajkumar Buyya "CloudAnalyst: A CloudSim-based Visual Modeller for Analysing Cloud Computing Environments and Applications"

[2]. M Vall O. M-Salem "Scaling Server Selection using a Multi-Broker Architecture”,IEEE 2003 (ICDCSW'03).

[3]. S K. Nair, S Porwal, Theo Dimitrakos, Ana Juan Ferrer, Johan Tordsson, Tabassum Sharif, Craig Sheridan,Muttukrishnan Rajarajan and Afnan Ullah Khan "Towards Secure Cloud Bursting, Brokerage and Aggregation" 2010 Eighth IEEE European Confrence on web services.

[4]. Stella Gatziu Grivas , Tripathi Uttam Kumar, Holger Wache "Coud Broker:Bringing Intelligence into the Cloud An Event-Based Approach"2010 IEEE $3^{\text {rd }}$ International Conference on Cloud Computing

[5]. Srikumar Venugopal, Rajkumar Buyya and Lyle Winton "A Grid Service Broker for Schedulling Distributed DataOriented Applications on Global Grids"

[6]. M. Armbrust, A. Fox, R. Griffith, A.D. Joseph, R. Katz, A. Konwinski, G. Lee, D. Patterson, A. Rabkin, I. Stoika, M. Zaharia " Above the Clouds: a Berkeley view of Cloud Computing” http://abovetheclouds.cs.berkeley.edu

[7]. Internet World Stats "Facebook" http://www.internetworldstats.com/facebook.htm

\section{Authors' Profiles}

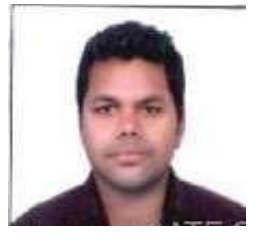

Ashish Sankla, New Delhi, 31 july 1986. M-tech in Computer Science Engineering, from University School of Information and Technology, Guru Gobind Singh Indraprastha University, Delhi. B-Tech in Computer Science Engineering from Guru Premsukh Memorial College of Engineering, Guru Gobind Singh Indraprastha University, Delhi.

He is working as Assistant Professor, CSE Dept., at G. B Pant Govt Engineering College, from January-2013. He qualified UGC-NET-2012 and scholar of his M-Tech batch.. 